\title{
ACTORES, CONTRATOS Y MECANISMOS DE PAGO: EL CASO DEL SISTEMA DE SALUD DE SALTA
}

Daniel Maceira*

Cintia Cejass

\section{Resumen}

Este estudio analiza la asociaciơn existente entre la estructura del mercado privado de servicios de salud, mecanismos de pagos a proveedores por parte del sistema de aseguramiento social, y respuesta en términos de organización interna y transferencia de riesgo en la provincia de Salta. Para ello se realizaron entrevistas en profundidad a actores claves y se relevaron indicadores de competencia, estrategias, contratos y mecanismos de pago en una muestra de prestadores públicos y privados de salud. Se concluye que un aumento en la escala poblacional incrementa el nivel de competencia entre prestadores y disminuye el poder relativo del principal competidor. A su vez, se desarrollan mayores incentivos a diferenciarse mediante estrategias diferentes a los precios como la reputación, hotelería e infraestructura.

* Ph.D. en Economia, Boston University, especializado en economia de la salud y organización industrial. Lic. en Economía, Universidad de Buenos Aires (UBA). Es Director del Programa de Salud del Centro de Implementación de Políticas Públicas para la Equidad y el Crecimiento (CIPPEC), Investigador Titular del Centro de Estudios de Estado y Sociedad (CEDES), e Investigador Adjunto del Consejo Nacional de Investigaciones Cientificas y Técnicas (CONICET), e.mail: dmaceira@cippec.ong.

"Lic. en Ciencias Políticas, Universidad Católica Argentina Maestría en Ciencias Sociales y Salud, (CEDES-FLACSO), tesis en curso. Coordinadora del Programa de Salud del Centro de Implementación de Políticas Públicas para la Equidad y el Crecimiento (CIPPEC)., e.maill:ccejas@cippec.org 
Clasificación JEL:I11,118

Palabras clove: servicio de salud - descentralización - mecanismos de pago en salud - mercado de salud - actores públicos y privados en salud

\begin{abstract}
Decentralization in health care strategy and goals definition, as well as in program implementation moves public discussion to the provincial level. This study analyzes the association among the market structure of the private health care services, payments mechanisms and responses related to internal organization and transference of risk between health providers and financiers in Salta. In depth interviews to key actors and a survey performed to a sample of public and private institutions allows to obtain information about perceived competition, strategies, contracts and payment mechanisms. The study finds a correlation between population scale, competition and relative bargaining power of the main competitor. This scheme friggers incentive to differentiated production using different strategies beyond prices, as reputation, product mix and infrastructure.
\end{abstract}

JEL Classiffcation: 111,118

Keywonds: health care service - decentralization - health care payment mechanism - health care market - public and private actors in health care

\title{
NTRODUCCION
}

Las estructuras de la oferta de servicios de salud (Hospitales, CAP, clínicas y sanatorios) y de su demanda (directamente por los pacientes o a través de sus seguros sociales o privados de salud) actúan como condicionantes del esquema de negociación y posterior implementación de mecanismos de pago, condicionando la calidad de atención, su sesgo preventivo, etc. A su vez, la estrategia de cada actor depende en última instancia de la estructura del mercado de servicios (número y distribución de demandantes y oferentes, grado de diferenciación de servicios, presencia de barreras a la entrada). 
El objetivo del presente documento es analizar la asociación existente entre la estructura del mercado proveedor de servicios de salud, los mecanismos de pagos a proveedores por parte del sistema de aseguramiento, y la respuesta en términos de organización interna y transferencia de riesgo en la provincia de Salta. La sección 2 sugiere un marco de referencia para el estudio de los mecanismos pago en salud y su aplicación en Argentina. En las secciones 3 y 4 se hace referencia al contexto en que se desarrollan estos mercados. Para ello se realiza una breve descripción socioeconómica y sanitaria de la provincia, una aproximación de la estructura del sistema sanitario en Argentina, y las particularidades que adquiere en la provincia de Salta.

A continuación se discuten los principales resultados del estudio de campo. En esta dirección, la sección 5 presenta las preguntas de investigación y se describe brevemente la metodología utilizada. Posteriormente se analiza el comportamiento de los financiadores y prestadores privados de servicios de salud, relevando motivos de competencia, estrategias para atract pacientes $y$ financiadores, distribución y fuentes de financiamiento. La sección 7 analiza los mecanismos de negociación de contratos y de pago. Finalmente el documento analiza el subsector público en la sección 8 y sus diferencias con el subsector privado, haciendo referencia a la organización del sistema, los diversos tipos de gestion de los hospitales, sus competencias y sus fuentes de financiamiento. Por último, las conclusiones se presentan en la sección 9 resumiendo las respuestas a las preguntas de investigación.

\section{MARCO TEORICO}

Los "mecanismos de pago" se refieren a la manera en que el dinero es asignado desde una fuente de recursos (una estructura gubernamental, una compañia de seguros u otro pagador) hacia una institución proveedora de servicios de salud (hospital, clínicas, laboratorios, farmacias, etc.) o hacia un proveedor individual (médico, enfermera, psicólogo, etc.). La configuración utilizada para la instrumentación de cada mecanismo de pago promueve un conjunto de incentivos en proveedores que impactan en el comportamiento de los mismos en términos de cantidad, calidad y tipos de servicios que brindan (Chemichovsky et al, 2003).

Un sistema de salud involucra a varios actores que interactuan entre sí y 
con los pacientes en estructuras habitualmente descentralizadas y donde es difficil de observar perfectamente la oportumidad y calidad de la atención. En este contexto, la diversidad de grupos de interés y el poder de negociación de cada uno de ellos se erige como condicionante del nivel y la estructura de pagos que finalmente será empleada en la relación contractual que una a los actores. De este modo, un. mecanismo de pago es, en cierta medida, producto del poder de negociación de las partes que interactúan en los "mercados de salud" (Maceira, 1998).

Las respuestas de los proveedores de servicios de salud a las diversas modalidades de pago han sido analizadas en la literatura económica principalmente a través de la teoria de contratos (Hart, O. y Holmstrong, B., 1987) surgida a partir de la denominada teoría de la agencia. La misma reconoce y modela explicitamente los conflictos potenciales de interés entre diferentes actores, enfatizando en la información asimétrica como principal obstáculo para monitorear esfuerzos en una relación contractual (en el caso de los sistemas de salud, refiere a la relación contractual entre financiadores y proveedores o entre pacientes y prestadores de servicios de salud).

La esencia de la teoria de la agencia radica en que un individuo u organización - el principal - contrata a otro individuo u organización - el agente para que realice un conjunto de acciones o sea el responsable de tomar ciertas decisiones. Sin embargo, existen obstaculos que impiden que dicha relación se desarrolle de la manera en la cual el principal la desea. Ello se atribuye a que los objetivos del agente pueden no estar alineados con aquellos del principal y, ante un escenario de información asimétrica, y monitoreo imperfecto, la parte más informada puede comportarse oportunísticamente.

Particularmente en los sistemas de salud, la presencia del "tencer pagador" (el seguro de salud contratado por el paciente) lleva a la identificación de dos principales (paciente y seguro) y un agente (el prestador de servicios). La teoría de la agencia se focaliza entonces, en el diseño de instrumentos que permitan minimizar dicha desarticulación. Entre ellos se encuentran, los mecanismos de selección de los agentes, el diseño de alternativas de monitoreo, y principalmente el más relevante en función del objeto del presente estudio, el diseño de los mecanismos de pago.

El diseño de un sistema de remuneración que aliñe los esfuerzos de los agentes con los objetivos del principal deberá basarse en dos componentes: el "nivel 
de pago" y la "estructura de pago". El "nivel" deberá ser igual o mayor a la compensación que el agente podría recibir en otras circunstancias similares (costo de oportunidad). La "estructura del pago" debe ser diseñada de manera que provea el más alto premio al agente por su esfuerzo, de modo de motivar la acción deseada del agente. En todo caso, este esquema de incentivos responde a factores estrictamente monetarios, más allá de las motivaciones no-monetarias que impulsan el accionar de los actores en el sistema de salud (solidaridad, compromiso social, prestigio, trabajo en equipo, etc.).

Cada método de pago presenta diferentes impactos en têrminos de eficiencia, calidad y equidad. Tradicionalmente los mecanismos de pago más utilizados han sido cuatro relativamente simples: capitación, pago por prestación, salarios (presupuesto si el pago se efectúa a una institución), y pago por diagnóstico o módulo.

1. Capitación. Bajo este sistema, los prestadores reciben un monto de dinero por cada persona enrolada, para cubrir un nivel especifico de cuidado de la salud y ofrecer un paquete definido de servicios, por un periodo de tiempo predeterminado. Es por lo tanto un mecanismo de pago de tipo prospectivo. Los provedores reciben un monto fjjo por paciente, y luego de descontar todos los costos del tratamiento, retienen el excedente como un ingreso propio. Es por ello, que el riesgo financiero del pagador es bajo y el del proveedor es alto. El pago por cápita induce a la eficiencia siempre que los recursos asignados se encuentren asociados con el tamat̃o de la población atendida y sus problemas sanitarios. Asf́, una cápita muy baja puede generar racionamiento y selección de riesgos por parte de los prestadores. A medida que el valor de la cápita incluya ajustes para perfiles epidemiologicos especificos el esquema se hace más equitativo.

2. Pago por prestación. Bajo este sistema los prestadores reciben un ingreso de acuerdo con el número y tipo de servicios brindados al paciente. Este es un sistema de pago retrospectivo. En este caso, el riesgo financiero lo asume el pagador, ya que debe efectuar desembolsos por cada prestación que le factura el prestador. Esto a su vez puede generar una tendencia de los proveedores a incrementar el número de servicios (demanda inducida), muchos de los cuales en ciertas ocasiones no son requeridos. Por este motivo, en ocasiones, el mecanismo de pago por prestación puede ser acompañado por techos de desembolso (presupuesto con techo prestacional).

3. Salarios. Bajo este sistema, los profesionales de la salud reciben una remuneración en función de una cantidad fija de horas trabajadas. Este 
esquema tiene su correlato en el presupuesto fijo bistónico de las instituciones sanitarias. Como tal, el monto del salario no guarda relación con el número de visitas, de la cantidad de pacientes, el número de casos, la severidad de los mismos, etc., lo que genera un incentivo a la subprestación de servicios, en tanto el ingreso neto se vincula con una reducción en los costos de operación. Asimismo, a menor el nivel de salario y presupuesto, mayor es el incentivo a reducir los costos operativos, profundizando el riesgo de subprestación.

4. Pago por diagnóstico. Consiste en un monto fijo que cubre todos los servicios asociados con un caso o enfermedad particular, y es aplicado de manera más frecuente para pacientes hospitalizados. La unidad de pago es menos agregada que en la cápita, aunque más desagregada que el pago por prestación.

La teoría del principal - agente argumenta que, dados los problemas de información imperfecta, aversión al riesgo, múltiples tareas y eficiencias asociadas al trabajo grupal, especialmente observadas en el mercado laboral de la salud (Robinson, 2001), para contrarrestar los efectos no deseados de los mecanismos de pago tradicionales, es necesario diseñar acuerdos de tipo "mixtos". Es por ello que para paliar la deficiencia que poseen los instrumentos anteriormente nombrados, se han diseîado algunas alternativas que buscan combinar elementos prospectivos y retrospectivos.

I. 1. Implementación de los mecanismos de pago: estructura del mercado, poder de negociación y racionalidad

De las características que presenta un contrato de salud se desprenderán un conjunto de incentivos con impacto en la cantidad y calidad de la utilización de los servicios médicos, la transferencia de riesgo entre actores y la eficiencia en la utilización de los recursos. La dirección de dichos efectos generalmente no resulta compatible entre actores. En tanto un financiador probablemente prefiera transferir el riesgo financiero al prestador a través de contratos capitados o presupuestos globales, el prestador preferirá un contrato por prestación, para no correr riesgos financieros al tiempo que optimice la calidad de los servicios que brinda. Es por ello que los contratos de salud se encontrarán en función, en primera instancia, del poder de negociación de las partes, y en segundo lugar, de la función objetivo que presenten los actores. 
La literatura económica, a través del enfoque tradicional de la Organización Industrial plantea como marco general de análisis el paradigma Estructura-Conducta-Desempeño. Según el mismo, desempeño en una industria - mercado particular (el logro de los objetivos propuestos) depende de la conducta de los demandantes y oferentes (dado por el comportamiento estratégico de los agentes que hacen parte de la oferta, como la política de fijación de precios, mecanismos de pago, publicidad, investigación y desarrollo, cooperación, fusión comercial, estrategias legales y demás). A su vez, la conducta depende en última instancia de la estructura del mercado, el cual se puede caracterizar por el número y distribución de los demandantes y oferentes, que depende a su vez, del tamaño de las empresas, el número de competidores y demandantes, el grado de diferenciación de los mismos, la presencia o no de barreras a la entrada y la estructura de costos.

Bajo esta línea de argumentación, el análisis de la estructura de la demanda y de la oferta de bienes y servicios de salud resulta fundamental dentro del esquema de negociación y posterior implementación de una estructura de pagos. En un sistema de salud, la oferta está compuesta por los prestadores de servicios de salud (hospitales, clínicas, laboratorios, farmacias, médicos particulares, etc.), mientras que la demanda, si bien en última instancia está representada por los pacientes, a los efectos del estudio de los mecanismos de pago, la misma puede considerarse conformada por los financiadores (obras sociales, prepagas, PAMI, etc). De esta manera, como el poder de negociación estará fuertemente condicionado por la estructura del mercado, resulta necesario caracterizar la misma (Maceira 1998). Indicadores que permiten describir la oferta y la demanda están dados por el grado de concentración de las instituciones, el tamaño de población que cubren, las estrategias de diferenciación de los productos que ofrecen, el nivel de integración. vertical de servicios, la posibilidad de llegar a acuerdos colusivos, etc. Una combinación de ellos determinará la capacidad de negociación entre sectores.

\section{CARACTERISTICAS SOCIOECONOMICAS $Y$ SANITARIAS DE SALTA}

La provincia de Salta está caracterizada por una significativa concentración de la población en pocos departamentos: la Capital provincial, San Martín y Orán concentran el $67 \%$ de la población en tanto quince de los veintitrés departamentos cuentan con poblaciones rurales superiores al $25 \%$ del total. Por 
estos motivos, gran parte de la población no cuenta con acceso a servicios básicos tales como agua de red o cloacas en sus hogares. Tales son los casos de los departamentos de Rivadavia, Santa Victoria, Molinos, San Carlos o Iruya.

Cuadro 1- Indicadores socio-demográficos (por departamento)

\begin{tabular}{|c|c|c|c|c|c|}
\hline Partido & $\begin{array}{c}\text { Poblectón en } \\
2001\end{array}$ & $\begin{array}{l}\text { Porcentaje de la } \\
\text { población provincial }\end{array}$ & $\begin{array}{l}\text { Porcentaje de } \\
\text { población con NBI }\end{array}$ & $\begin{array}{c}\text { Porcentele de poblackón sin } \\
\text { cobertura }\end{array}$ & $\begin{array}{l}\text { Porcentaje de } \\
\text { pobleción nural }\end{array}$ \\
\hline Anta & 49.841 & 4,6 & 43,9 & 67,1 & 36,6 \\
\hline Cechl & 7.280 & 0,7 & 36,2 & 75,8 & 69,8 \\
\hline Cafayate & 11.785 & 1,1 & 27,4 & 59,5 & 9,1 \\
\hline $\begin{array}{l}\text { Cerrillos } \\
\text { Chicosana }\end{array}$ & $\begin{array}{l}26.320 \\
18.248\end{array}$ & $\begin{array}{l}2,4 \\
1,7\end{array}$ & $\begin{array}{l}35,6 \\
34,9\end{array}$ & $\begin{array}{l}55,5 \\
56,8\end{array}$ & $\begin{array}{l}37,8 \\
35,7\end{array}$ \\
\hline General Guemes (Salta) & 42.255 & 3,9 & 31,4 & 64,1 & 10,8 \\
\hline General José de San Martin & 139.204 & 12,9 & 40,7 & 71,8 & 18,6 \\
\hline Guachlpes & 3.211 & 0,3 & 44,7 & 60,8 & 100,0 \\
\hline Iruya & 6.368 & 0,6 & 49,7 & 79,9 & 100,0 \\
\hline La Caldera & 5.711 & 0,5 & 32,8 & 58,4 & 47,8 \\
\hline La Candelarla & 5.286 & 0,5 & 35,1 & 63,9 & 55,9 \\
\hline La Poma & 1.735 & 0,2 & 43,4 & 68,0 & 100,0 \\
\hline La VIกต & 7.152 & 0,7 & 34,1 & 59,9 & 52,9 \\
\hline Los Andes & 5.630 & 0,5 & 41,9 & 66,3 & 24,1 \\
\hline Metan & 39.006 & 3,6 & 30,8 & 58,2 & 18,4 \\
\hline $\begin{array}{l}\text { Mollnos } \\
\text { Oran }\end{array}$ & $\begin{array}{r}5.565 \\
124.029\end{array}$ & $\stackrel{0,5}{11,5}$ & $\begin{array}{l}48,4 \\
43,2\end{array}$ & $\begin{array}{l}75,2 \\
67,3\end{array}$ & $\begin{array}{l}100,0 \\
10,7\end{array}$ \\
\hline $\begin{array}{l}\text { Rlvadavla } \\
\text { Rosario de la Frontera }\end{array}$ & $\begin{array}{l}27.370 \\
28.013\end{array}$ & $\begin{array}{l}2,5 \\
2,6\end{array}$ & $\begin{array}{l}65,5 \\
26,6\end{array}$ & $\begin{array}{l}86,7 \\
63,0\end{array}$ & $\begin{array}{l}86,6 \\
20,7\end{array}$ \\
\hline Rosarlo de Lerma & 33.741 & 3,1 & 31,2 & 57,2 & 25,5 \\
\hline Selta Cepltel & 472.971 & 43,8 & 21,3 & 51,9 & 0,8 \\
\hline San Carlos & 7208 & 0,7 & 35,3 & 72,6 & 100,0 \\
\hline Santa Victoria & 11.122 & 1,0 & 56,7 & 82,6 & 100,0 \\
\hline Total Provincle & 1.079 .051 & 100,0 & & & \\
\hline
\end{tabular}

Fuente: Censo Nacional 2001

Paralelamente, se registra una relación positiva entre el porcentaje de hogares con NBI y la proporción de su población que vive en el campo. De la misma manera, la vinculación entre NBI y ausencia de cobertura sanitaria también es positiva. De este modo, la población en situación de mayor vulnerabilidad social en la provincia se encuentra ubicada en zonas rurales de baja densidad y cobertura en salud. 
Gráfico 2 - Salta. Relación entre NBI y ausencia de cobertura sanitaria (Por departamento)

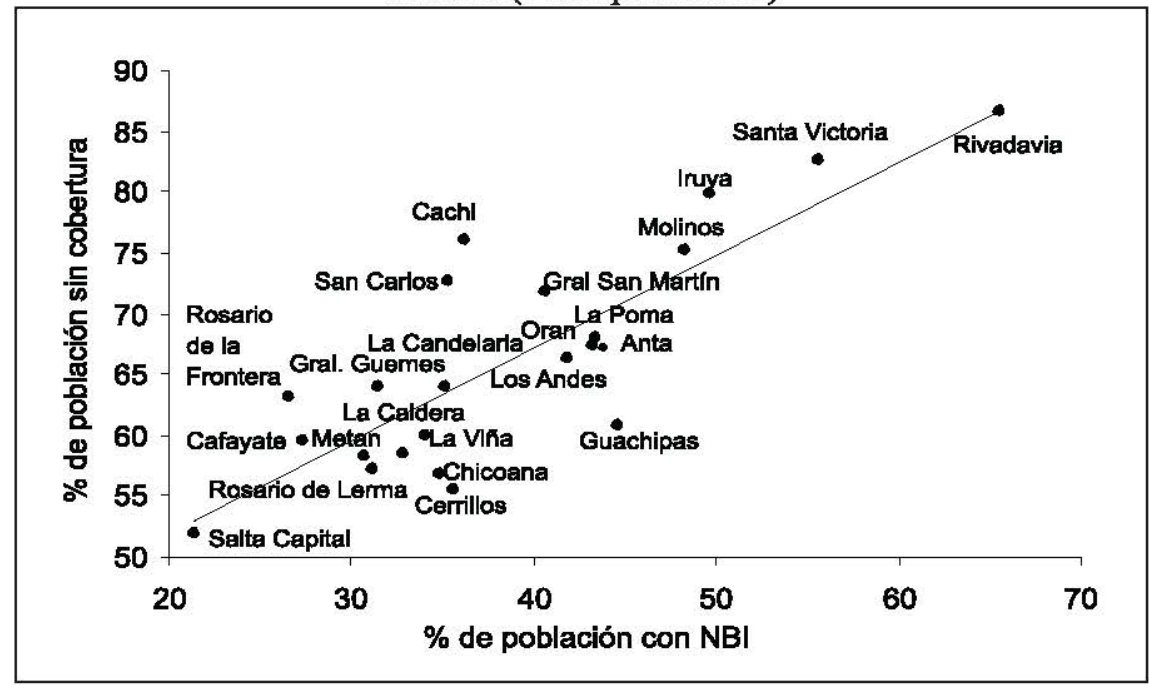

Fuente: Censo Nacional 2001

A partir de la información sobre gasto provincial por finalidad, publicados por la Dirección Nacional de Coordinación Fiscal con las Provincias, se observa que la provincia de Salta es la segunda jurisdicción del país en términos de la proporción del gasto destinada a salud sobre el total. El mismo que representa un $20,61 \%$, sólo superado por la Ciudad de Buenos Aires que destina a salud un $30,62 \%$ de su gasto total. En términos del gasto en salud por habitante (Maceira, 2010), Salta se encuentra cerca del promedio nacional con un total de \$305 por habitante en 2005, lejos de los \$1106 de Santa Cruz (la de mayor gasto per cápita en salud) pero un 57\% superior a los \$194 de Corrientes (la de menor gasto per cápita en salud).

\section{ESTRUCTURA DEL SISTEMA DE SALUD DE SALTAY SUS PRINCIPALES ACTORES}

La cobertura de servicios de salud en la Argentina se encuentra compartida 
entre el sector público, el sector de las obras sociales y el sector privado, con una fuerte interrelación tanto a nivel de la provisión de servicios como en términos de financiamiento. Considerando la complejidad de las relaciones, se propone distinguir entre fuentes de financiamiento, financiadores y prestadores de servicios de salud (Maceira, 2006).

Gráfico 3 - Estructura de financiamiento, aseguramiento y provisión de servicios del sistema de salud. Provincia de Salta.

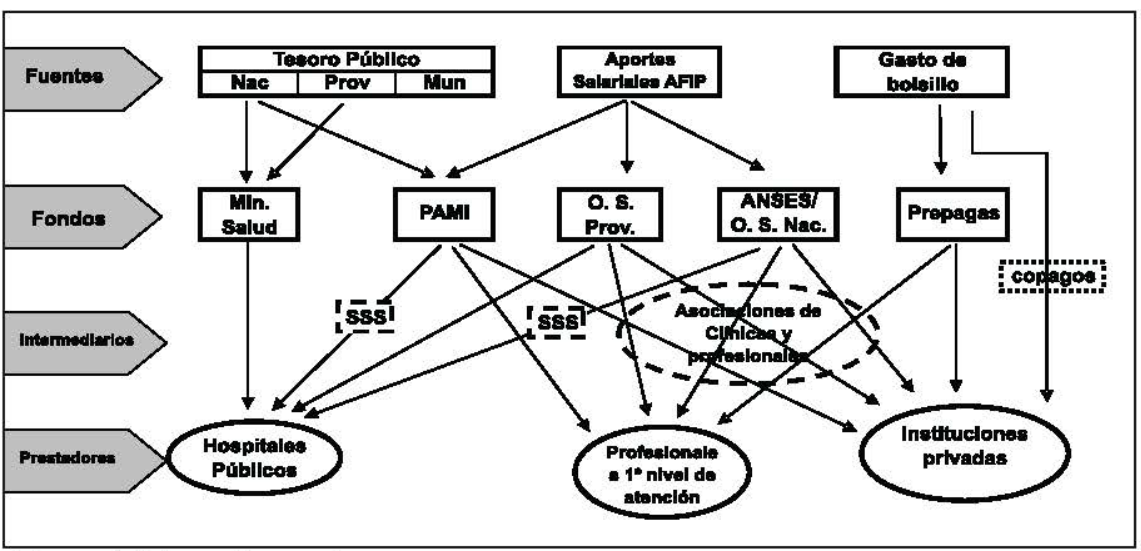

Fuente: Elaboración propia

Las fuentes de financiamiento del sistema de salud provincial se pueden agrupar en tres grandes bloques, en paralelo con el modelo nacional. En el sector público es el Tesoro provincial, compuesto por ingresos tributarios y transferencias nacionales, directas o en especies, el cual asigna sus recursos al Ministerio de Salud. Asimismo, el sector de la seguridad social se financia con los aportes salariales y las contribuciones patronales asociadas al trabajo autónomo y en relación de dependencia de empleados públicos (dirigidas a la obra social provincial) o privado (obras sociales nacionales). Finalmente el gasto de bolsillo está compuesto por los aportes directos de los pacientes a los servicios de salud, prepagas, copagos, medicamentos, etc.

El PAMI, por su parte, brinda cobertura al $11,8 \%$ de la población provincial. Su financiamiento proviene de aportes de los trabajadores autónomos en actividad, del personal en actividad, la contribución de los pasivos que varía entre un 3\% y $6 \%$, según superen o no el haber mínimo; y los aportes del tesoro nacional 
que determina la Ley de Presupuesto Nacional anualmente. El subsector público de Salta, a través de su red de centros asistenciales, brinda asistencia al $60.4 \%$ de la población. Esto es 651.995 personas que no poseen cobertura de obras sociales ni prepagas'. De acuerdo con la guia de establecimientos (MinSalud, 2000) actualizada mediante entrevistas personalizadas para esta investigación, el sector público cuenta con 50 establecimientos con internación y 467 centros de atención. primaria.

La provincia de Salta ha sido histónicamente una pionera en reformas de su sistema de salud, desde el modelo de atención primaria desarrollado durante las ultimas décadas hasta el lanzamiento de Seguros Provinciales de Salud con gestión departamental y modelos de autogerenciamiento hospitalario, administración mixta y tercerización.

Uno de los principales financiadores del sistema de salud de Salta, es la obra social provincial denominada Instituto Provincial de Salud de Salta (IPS), definido por la ley 7127 del 2001 como una entidad autárquica con personería jurídica dotada de individualidad administrativa, económica y financiera. Actualmente brinda cobertura a 201.836 personas, el $18.7 \%$ de la población de Salta ${ }^{2}$ La afiliación es obligatoria para todos los empleados de la administración pública provincial y ranicipal; los jubilados y pensionados del sistema de previsión social de la Provincia, y el personal temporario y contratado que cumpla funciones en organismos provinciales y municipales. También puede captar afiliados adherentes voluntarios. El IPS concentra sus actividades en la administración de los servicios de salud que brinda a sus afiliados, a través de prestadores contratados y/o mediante sus servicios propios de farmacia y optica.

En esta dirección, mantiene relación contractual con establecimientos asistenciales públicos y privados, tanto de capital como del interior de la provincia. De esta manera brinda servicios médicos en todas las especialidades, sin cobro de arancel diferenciado. Los afiliados tienen cubierto el $80 \%$ del valor de las consultas y prácticas, en tanto el otro $20 \%$ se encuentra a cargo del afiliado (coseguro). En algunos casos, la Asociación de trabajadores de la Sanidad -ATSA- y Unión del Personal Civil de la Nación -UPCN- actuan como reaseguros, cubriendo dicho coseguro mediante una cuota fija de sus afiliados. En cuanto a los medicanentos registrados en el vademécum de la institución, éstos están cubiertos en un $40 \%$ por la obra social y el otro $60 \%$ a cargo del afiliado.

${ }^{1} \mathrm{NNDEC}, 2001$

${ }^{2}$ Fuente: Instituto Provincial de Salud. Población beneficiaria al 31/05/2004 
Por su parte, el PAMI, es la institución de aseguramiento social más importante del país que brinda servicios a 3.200 .000 de personas mediante sus 36 "Unidades de Gestión Local". A la jurisdicción de Salta le corresponde la número XII, contando con 59.402 afiliados $^{3}$, un $5.5 \%$ del total de la población provincial y un $17.09 \%$ de los que cuentan con algún tipo de cobertura de obra social nacional ${ }^{4}$.

Finalmente, las obras sociales sindicales, reguladas por la Superintendencia de Servicios de Salud con competencia nacional, brindan cobertura a 288.228 afiliados en la provincia de Salta. La obra social de Personal Rural y Estibadores, en primer lugar, así como la del personal de la Construcción y de Empleados de Comercio y Actividades Civiles, en segundo término, son las que mayor cantidad de afiliados agrupan cubriendo entre ambas el $40 \%$ del total del rubro. Asimismo, siete obras sociales nacionales cubren el $60 \%$ del total de los beneficiarios de su grupo. El Gráfico 4 compara el peso relativo de cada uno de los financiadores de acuerdo al número de afiliados.

Gráfico 4 - Distribución del aseguramiento en Salta

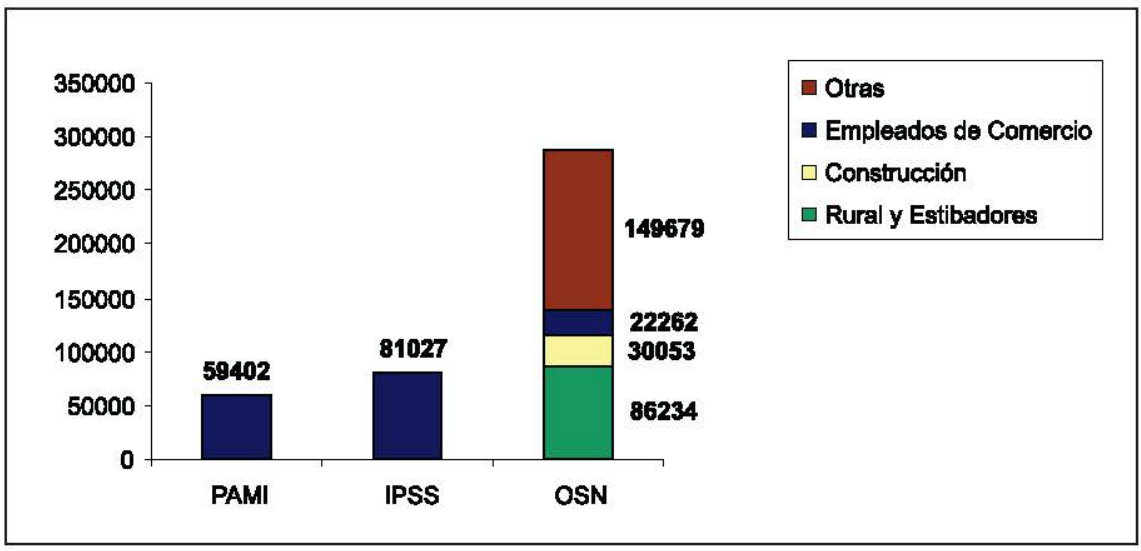

Fuente: Elaboración propia sobre la base de datos de superintendencia de servicios de salud y del Instituto Provincial de Salud

Por último, la provincia de Salta cuenta con 225 establecimientos privados, entre clínicas, sanatorios y centros de diagnóstico que incluyen

${ }^{3}$ Superintendencia de Servicios de Salud. 2006-200.

${ }^{4}$ Superintendencia de Servicios de Salud. Afiliados a Obras Sociales Nacionales. 2006-2007 
aproximadamente 80 laboratorios ${ }^{5}$. Los prestadores en su mayoria, se agrupan en entidades intermedias que permiten establecer contratos y negociar precios ante financiadores o pagos de deudas de una manera más efectiva. Las principales entidades en las zonas seleccionadas para este estudio son:

- Asociación de Clínicas y Sanatorios Privados de la Provincia de Salta (ACLISASA) que agrupa a la Clinica San Rafael, Maternidad Privada Salta S.A, Sanatorio San Roque S.A, Clínica del Niño S.R.L, Sanatorio Modelo S.A, Clínica Cruz Azul S.A, Clínica del Neurodignóstico, Clínica Luis Güemes, Clínica San Antonio (Tartagal), Clínica Privada Sagrado Corazón (Orán)

- Camara de Empresas Médicas formada por el Hospital Privado Santa Clara de Asis, Sanatorio El Parque y Sanatorio El Carmen.

- Asociación de Clinicas del Interior integrada por la Clínica Divina Misericordia, Clinica San Antonio, Clinica Mayo, Clinica Lourdes, Clínica San Lucas, Centro de Especialidades Médicas, Clínica Alem, Clínica Itati, Clínica Huachana, Clínica Metán y Clínica 9 de Julio.

- Cámara del Norte compuesta por la Clinica San Antonio (Tartagal), Clinica Privada Sagrado Corazón (Orán), entre otras. Conformada para brindar servicios a la Obra Social provincial

- Consorcio de Prestadores Privados de Salud de Salta (COPRESA), creado a principios de 2007 y conformado por la Asociación de Clinicas y Sanatorios Privados de la Provincia de Salta, la Cámara de Empresas Médicas de Salta y el Círculo Médico de Salta. Su función es la negociación conjunta del convenio integral con el Instituto Provincial de Salud de Salud de Salta-IPS, y gradualmente los convenios con las Obras Sociales nacionales.

Paralelamente, existen al momento de realizar esta investigación, tres instituciones que no se encuentran asociadas y son el Centro Asistencial de Salud CENESA, Hospital Privado Tres Cerritos y Clínica La Merced. Finalmente, existen círculos de profesionales de la salud entre los cuales se destaca el Círculo Médico de la ciudad de Salta que agrupa a unos 1.200 médicos de la ciudad capital.

\section{PREGUNTAS DE INVESTIGACION Y METODOLOGIA}

El objetivo de este estudio es analizar la asociación existente entre la

${ }^{s}$ Gúa de Establecimientos de Salud. Ministerio de Salud. 2000 
estructura del mercado proveedor de servicios de salud, los mecanismos de pagos a proveedores por parte del sistema de aseguramiento social, y la respuesta en términos de organización interna y transferencia de riesgo en la provincia de Salta. A partir de ello, las preguntas de investigación que serán desarrolladas en el presente capítulo son las siguientes:

1- Bajo el argumento de que los mercados más desarrollados en términos de escala poblacional generan un escenario de mayor nivel de competencia y la consecuente pérdida de poder relativo de sus prestadores, las preguntas que se desprenden de esta primera hipótesis son:

¿Qué características posee la estructura del subsistema privado de servicios de salud en los departamentos seleccionados de la provincia de Salta?; ¿Cuál es la percepción de los prestadores de servicios de salud entrevistados acerca del lugar que ocupa el lider en el mercado?; ¿Cómo se posicionan ellos mismos en el mercado?; ¿Disminuye la participación del líder a medida que aumenta la cantidad de competidores?; ¿Cuál es el nivel de competencia percibida por los prestadores?

2- En la medida on que aumenta ol tamaño del mercado y existen parámetros de competencia diferenciados distintos a los precios, las preguntas que surgen son: ¿Cuáles son los mecanismos que determinan la competencia en el sector salud de Salta?; ¿Qué lugar ocupan los precios y los contratos como mecanismos de competencia vis a vis variables de calidad de servicios percibida?

3- Los prestadores definen sus estrategias con respecto a sus dos principales; los financiadores y pacientes, de acuerdo a lo que perciben como importante para ellos.

En este sentido, ¿Cómo percibe el agente las diferencias entre sus dos principales?; ¿Cuáles son las estrategias desarrolladas por los prestadores para atraer pacientes y cuáles para atraer financiadores?; $¿$ Se diferencian entre sí?; $\measuredangle Q u e ́$ implicancias tiene su diferenciación o no diferenciación?

4- Considerando la existencia de financiadores con diferente peso relativo en el sistema sanitario de Salta: ¿Existe homogeneidad de cobertura entre las jurisdicciones?; ¿Quién define las características del contrato? 
5- A fin de evitar la situación de asimetría entre los prestadores y sus financiadores, los primeros se agrupan. En esta dirección las preguntas son: ¿Qué capacidad de negociación tienen las Asociaciones frente a los financiadores?; ¿Cuales son los incentivos para asociarse? y a partir de ello, Łexiste transferencia de riesgo de los financiadores a los prestadores?

6- Finalmente, y desde la perspectiva de política pública ¿Cuales son las características del subsistema estatal de provisión de servicios de salud que lo diferencia del subsector privado?

El análisis del caso de Salta consta de dos etapas metodológicas. La primera de carácter cualitativo consistió en un mapeo de actores con el objetivo de comprender la dinámica del sector salud en sus ámbitos público y privado. Para ello, se identificaron los principales referentes del sector de la provincia: financiadores del sistema y Asociaciones intermedias de profesionales y establecimientos. Entre ellos se contactó a autoridades del Ministerio de Salud de la provincia, de la Obra Social provincial -Instituto Provincial de Salud-, de la Unidad de Gestión Local de Salta del PAMI, de la Asociación de Clínicas y Sanatorios de Salta (ACLISASA), de laAsociación de Clínicas del Interior y del Circulo Médico de Salta.

Se diseñó un cuestionario para orientar las entrevistas en profundidad con los actores clave seleccionados. El instrumento relevó la percepción de los actores sobre características generales del mercado de salud local, focalizando en los objetivos y estrategias de los principales actores ( $f$ in a n ci a d ores, prestadores $y$ entidades intermedias); la estructura actual y la existencia de cambios a lo largo del tiempo en lo que respecta al marco normativo y legal, contratos $y$ mecanismos de pagos a

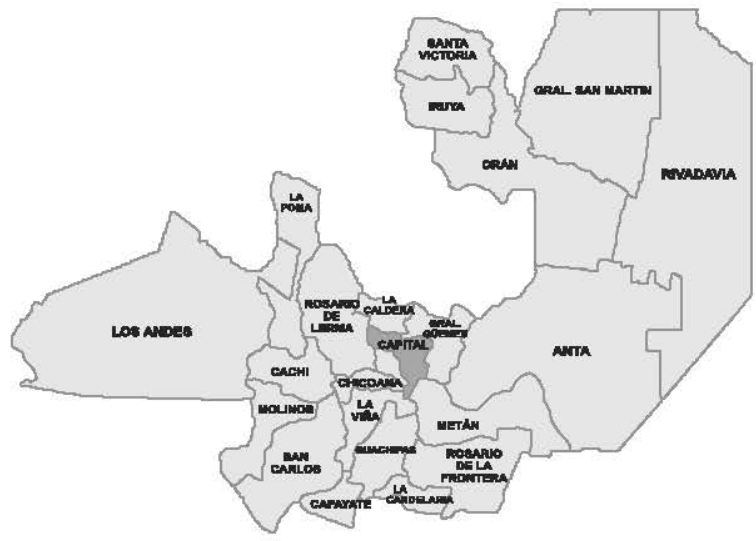
los proveedores de servicios; y finalmente, el impacto de estos cambios en la organización interna y en el financiamiento de las entidades financiadoras y proveedoras. 
La segunda etapa metodológica implicó en la selección de los departamentos que conformaron la muestra a prestadores públicos y privados. Debido a la imposibilidad de cubrir toda la provincia, se seleccionaron tres departamentos en base a criterios predefinidos (cantidad de población y, oferta privada y pública de establecimientos de salud polivalentes con internación). Sobre la base de esto criterios, los departamentos seleccionados de la provincia de Salta fueron San Martin, Anta y Capital. Finalmente, dentro de cada departamento se definió una muestra de los establecimientos a encuestar.

Para ello, se diseñó un cuestionario que relevó la percepción de los prestadores sobre las características generales del mercado de salud local, focalizando en sus objetivos y estrategias. Se indagó asimismo sobre la estructura actual y la existencia de cambios a lo largo del tiempo en el marco normativo y legal, contratos y mecanismos de pagos a los proveedores de servicios.

Se realizaron 21 entrevistas aplicando cuestionarios diferenciados a prestadores públicos y privados. Del total de los establecimientos privados polivalentes y con internación de la provincia de Salta se encuestó al 57,69\%, mientras que se realizó lo propio con el $12 \%$ de los establecimientos públicos. Un detalle por departamento se presenta en el siguiente cuadro. 
Cuadro 2 - Establecimientos públicos y privados seleccionados

\begin{tabular}{|c|c|c|c|c|c|c|c|c|c|}
\hline \multirow{3}{*}{ Depondancie } & \multicolumn{9}{|c|}{ Depertamentos } \\
\hline & \multicolumn{3}{|c|}{ Capital } & \multicolumn{3}{|c|}{ San Marth } & \multicolumn{3}{|r|}{ Ants } \\
\hline & $\begin{array}{l}N^{N} \text { total de } \\
\text { Esteblec. }\end{array}$ & $\begin{array}{l}\text { No Egtablec. } \\
\text { Enculastados } \\
\text { (2) }\end{array}$ & $\begin{array}{l}x \\
(2)\end{array}$ & $\begin{array}{l}N^{0} \text { total de } \\
\text { Establec. } \\
\text { (1) }\end{array}$ & $\begin{array}{c}N^{0} \text { Egtablec. } \\
\text { Encuestades } \\
\text { (2) }\end{array}$ & $\begin{array}{l}\% \\
(2)\end{array}$ & $\begin{array}{l}\mathrm{N}^{\mathrm{N}} \text { total de } \\
\text { Establec. } \\
\text { (1) }\end{array}$ & $\begin{array}{l}\text { NP Egtablec. } \\
\text { Enculestados } \\
\text { (2) }\end{array}$ & $\begin{array}{l}\% \\
\text { (2) }\end{array}$ \\
\hline Públlcos & $7(3)$ & 3 & $43 \%$ & 5 & 1 & $20 \%$ & 4 & 2 & $50 \%$ \\
\hline Prtvados & 13 (4) & 12 & $92 \%$ & 4 & 2 & $50 \%$ & 2 & 1 & $50 \%$ \\
\hline
\end{tabular}

Fuente: Elaboración propia

Cuadro 3 - Características generales de las instituciones encuestadas

\begin{tabular}{|c|c|c|c|c|c|c|c|c|c|}
\hline & \multicolumn{3}{|c|}{ 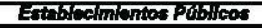 } & \multicolumn{3}{|c|}{ Establectinianos Privados } & \multicolumn{3}{|c|}{ TOED } \\
\hline Vurlable | Estadistico & Modka & $\operatorname{Max}$ & mn & Mockin & $\operatorname{Max}$ & Min & Wedie & $\operatorname{Mex}$ & Mh \\
\hline $\min ^{2}$ & 9008,07 & 14000 & 5000 & 2384,40 & 4500 & 1000 & $4065, \infty 00$ & 14000 & 1000 \\
\hline Po do cames & 162,43 & 400 & 40 & 53,63 & 110 & 20 & $\$ 8,00$ & 400 & 20 \\
\hline $\begin{array}{l}\text { Pr do consiobs: promedto } \\
\text { mentualue (I) }\end{array}$ & 19738,67 & 80000 & 1738 & 1406,82 & 3000 & 300 & 8147,00 & 80000 & 300 \\
\hline 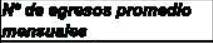 & 1835,00 & 6900 & 197 & 224,25 & 460 & 16 & 781,00 & 6800 & 16 \\
\hline Promedto difes ceme & 4,70 & 7,5 & 3,4 & 3,58 & 10 & 2 & 3,82 & 10 & 2 \\
\hline
\end{tabular}

Fuente: Elaboración propia sobre la base de encuestas de campo (2007).

(1) Para los públicos: no incluye las consultas por guardia.

Nota: Los medias fueron calculadas sobre la base de las respuestas positivas y no sobre el total de casos.

De acuerdo con lo relevado en la muestra de oferentes, el promedio de superficie cubierta de las instituciones públicas supera en 4 veces a la de los privados, siendo la dispersión entre éstos últimos (4.5) más amplia que la de los primeros (2.8). Respecto a la cantidad de camas, el sector público también supera en casi 3 veces al privado, aunque la mayor dispersión se registra entre los establecimientos públicos con una diferencia de 10 veces a 1 entre extremos.

En términos de consultas (Cuadro 3), la relación es de 13 a 1 entre los establecimientos públicos y los privados, sin embargo en términos de egresos de internación la relación sólo alcanza 8.1. En cuanto al promedio de días cama, el sector público supera al privado en 1.12 días, aunque la dispersion en el segundo es mucho mayor que en el primero. Dentro del sector privado, las clínicas que superan el promedio de días cama son aquellas que cuentan con convenios con PAMI, lo que requiere jornadas de internación promedio más elevadas. 


\section{COMPORTAMIENTO DE LOS ACTORES EN EL MERCADO}

Con el objetivo de analizar la conducta de mercado de los proveedores de servicios de salud y su estrategia frente a la competencia y ante los financiadores, se relevaron indicadores que reflejan la percepción de los proveedores privados de servicios sobre la morfología del mercado en el que actúan, dado que a partir de dichas percepciones se desarrollan sus planes de acción.

Cuadro 4 - Estructura percibida del mercado

\begin{tabular}{|c|c|c|c|c|c|c|c|c|c|}
\hline & \multicolumn{3}{|c|}{$\begin{array}{l}N^{P} \text { de proovedores que } \\
\text { considera competidores }\end{array}$} & \multicolumn{3}{|c|}{$\begin{array}{c}\text { Posición en el mercado de la } \\
\text { institución lider (\%) }\end{array}$} & \multicolumn{3}{|c|}{$\begin{array}{l}\text { Posicion en of mercado de la } \\
\text { institucion encuestada (\%) }\end{array}$} \\
\hline & Medla & $\operatorname{Max}$ & $M n$ & Madla & $\operatorname{Max}$ & $\mathrm{m} / \mathrm{n}$ & Media & $\operatorname{Max}$ & $\min$ \\
\hline Total & 4,64 & 13 & 0 & 50,00 & 100 & 20 & 35,00 & 100 & 16 \\
\hline Sofftes & 5,55 & 13 & 2 & 37,50 & 50 & 20 & 32,50 & 50 & 16 \\
\hline San Martin & 2,00 & 2 & 2 & 67,50 & 75 & 60 & 52,50 & 75 & 30 \\
\hline Ante & 0,00 & 0 & 0 & 100,00 & 100 & 100 & 100,00 & 100 & 100 \\
\hline
\end{tabular}

Fuente: Elaboración propia sobre la base de encuestas de campo (2007).

A partir de la información recogida mediante encuestas a los prestadores privados, la Tabla 4 reporta la percepción de la competencia y de participación relativa en la cobertura de servicios por parte de cada una de ellos. Se observa un número relativamente moderado de competidores, con un promedio de 4,64 para el total provincial. El departamento de Salta es aquel con mayor competencia percibida, $(5,55)$, mientras que en San Martín es de 2 y en Anta no se registró competencia, ya que los 2 proveedores de servicios de esa área declaran que no compiten entre sí, habiendo logrado una suerte de acuerdo para la recepción de la demanda.

Respecto a la percepción de la posición que ocupa el líder en términos de ingresos monetarios, en la capital provincial, ese porcentaje es del $37,50 \%$, lo cual demuestra la presencia de varios liderazgos que compiten entre sí. En San Martín, tal porcentaje alcanza el 67,50\% verificándose la presencia de un líder y en Anta al no generarse una competencia, ambas instituciones se posicionan como líderes. Respecto a cómo se perciben a sí mismas las instituciones encuestadas y qué porcentaje de los ingresos del mercado poseen, nuevamente Salta capital muestra un mayor tamaño de mercado que el resto, donde la competencia es mayor y la concentración y la porción del mercado que ocupa cada uno es relativamente más 
baja. Del departamento de Anta, el promedio del mercado que manifestó poseer la institución encuestada es levemente menor respecto al que ocupa el lider. Esto podría alentar la creencia de una gran diferenciación de servicios, y por lo tanto mayor poder relativo, para definir condiciones de contratación y oferta.

El Gráfico 5 confirma la percepción del nivel de competencia de las instituciones encuestadas. Mientras que en Salta un $18 \%$ considera que el nivel de competencia es alto, seguramente marcado por las instituciones líderes, un $45 \%$ considera que es medio y un $36 \%$ que es bajo. En San Martín, el nivel de competencia percibido es relativamente medio y en Anta no se percibe competencia. De este modo se confima una de las hipótesis de trabajo que sostiene una relación directa entre tamaño de mercado, medido en términos de población con mayor competencia (número de prestadores y participacion de cada uno), y los incentivos a diferenciarse.

Por su parte, el Gráfico 6 indica cuáles son los motivos de competencia percibidos por los encuestados (expresados como porcentaje del total de entrevistados que eligieron cada opción como relevante). Reputación (63\%), hotelería e infraestructura ( $42 \%$ ) son los más reconocidos, mientras que los precios (4\%) y la definición de contratos $(4 \%)$ no son considerados relevantes como fuentes de competencia. Esto puede atribuirse a que la definición de precios y contratos una vez negociados, dejan de ser una variable de diferenciación entre instituciones. De forma contraria, si el aseguramiento de la demanda dismimuyera y los pacientes abonaran las consultas directamente de su bolsillo, la variable "precio" de los servicios deberia determinar la elección entre prestadores. 
Gráfico 5 - Nivel de Competencia

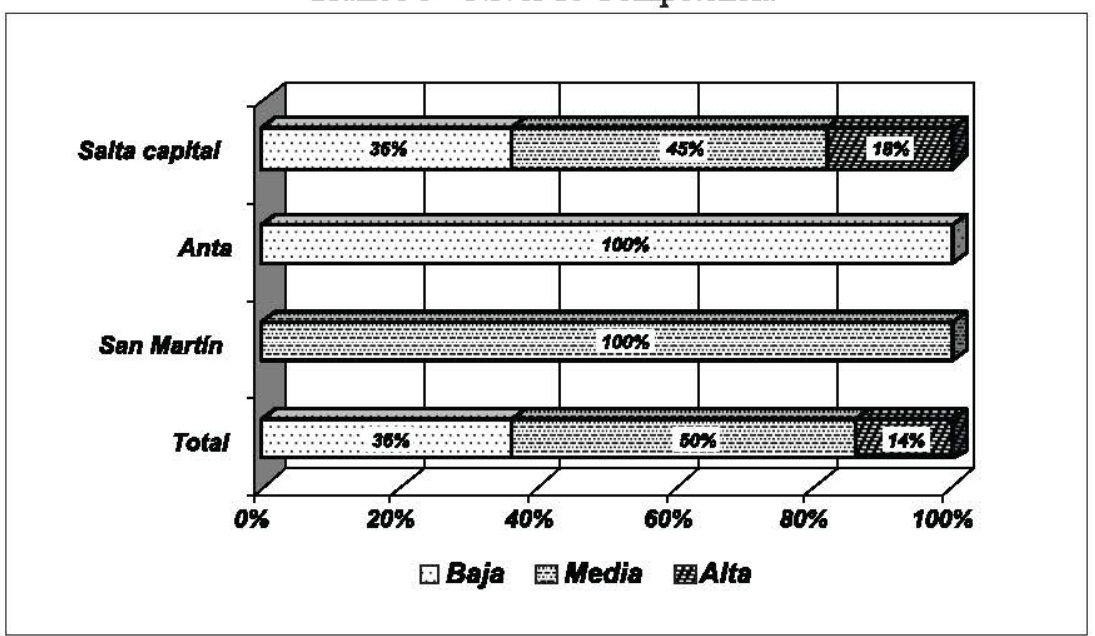

Fuente: Elaboración propia sobre la base de encuestas de campo (2007).

Gráfico 6 - Motivos de competencia

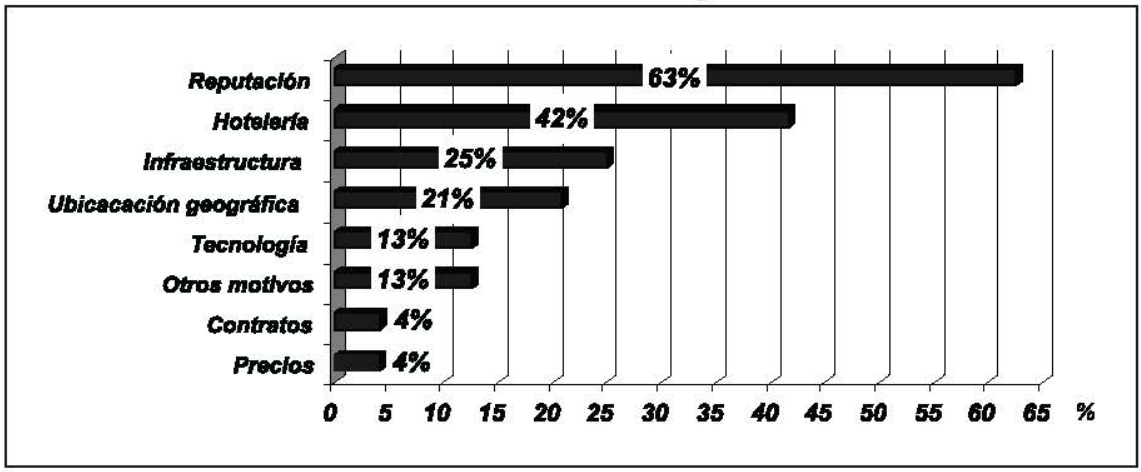

Fuente: Elaboración propia sobre la base de encuestas de campo (2007).

El Cuadro 5 muestra para los tres departamentos seleccionados, el peso relativo de cada sistema de aseguramiento/pago en el ingreso total de las instituciones, el promedio departamental y total, con sus valores mínimos y máximos. Mientras que en Salta Capital y en Anta, el principal financiador es la 
Obra Social provincial, con una participación promedio del $34,18 \%$ y $30 \%$ respectivamente en el presupuesto de los prestadores, en San Martín ocupa este lugar el PAMI con una participación promedio del $50 \%$.

De los 15 prestadores entrevistados, 6 manifestaron recibir aportes de PAMI, de los cuales 3 pertenecen al interior de la provincia. Si bien la participación de esta institucion en el presupuesto de las entidades de la capital provincial es relativamente bajo, al analizar los valores máximos se destaca la existencia de al menos un prestador cuyo presupuesto depende en un $85 \%$ de su aporte. En San Martín, las 2 clínicas entrevistadas también dependen de los aportes del PAMI aunque en proporciones muy diferentes: $80 \%$ y $20 \%$ respectivamente. Catorce entrevistados reciben aportes del IPS, convirtiéndose en el fondo de aseguramiento de mayor participación promedio en el presupuesto de los prestadores. En Salta Capital alcanza un máximo de $75 \%$, con un minimo de $5 \%$. Tanto en San Martín corno en Anta, con menor participación del empleo público, su incidencia es menor.

Las Obras Sociales Nacionales proveen financiamiento a 13 de los 15 establecimientos y se ubican en segundo lugar en cuanto a su participación en el financiamiento del sistema, aunque en ningún caso superan al $55 \%$ de los recursos recibidos por las instituciones. Por su parte, las prepagas brindan financiamiento a 10 de las 15 instituciones y adquieren mayor presencia en la capital con un $17,82 \%$ de participación en el financiamiento. Finalmente, la contratación del sector privado por parte del Estado provincial no es significativa y ol aporte de los particulares es marginal. 
Cuadro 5 - Participación relativa (en \%) de los fondos de aseguramiento en el financiamiento institucional

\begin{tabular}{|l|c|c|c|c|c|c|c|c|c|c|c|c|}
\hline \multirow{2}{*}{ Financiadores } & \multicolumn{3}{|c|}{$\begin{array}{c}\text { Salta } \\
\text { (11 casos) }\end{array}$} & \multicolumn{3}{c|}{$\begin{array}{c}\text { San Martín } \\
\text { (2 casos) }\end{array}$} & \multicolumn{3}{c|}{$\begin{array}{c}\text { Anta } \\
\text { (1 caso) }\end{array}$} & \multicolumn{3}{c|}{ Total } \\
\cline { 2 - 13 } & Media & Máx. & Mín. & Media & Máx. Mín. & Media & Máx & Mín. Media & Máx. Min \\
\hline PAMI (6) & 11,82 & 85 & 0 & 50 & 80 & 20 & 25 & 25 & 25 & 18,21 & 85 & 0 \\
\hline $\begin{array}{l}\text { Obra Social } \\
\text { Pcial (14) }\end{array}$ & 34,18 & 75 & 5 & 25 & 40 & 10 & 30 & 30 & 30 & 32,57 & 75 & 5 \\
\hline $\begin{array}{l}\text { Obras Sociales } \\
\text { Nacionales } \\
\text { (13) }\end{array}$ & 30,73 & 55 & 0 & 20 & 30 & 10 & 25 & 25 & 25 & 28,79 & 55 & 0 \\
\hline Prepagas (10) & 17,82 & 55 & 0 & 2,5 & 5 & 0 & 0 & 0 & 0 & 14,36 & 55 & 0 \\
\hline $\begin{array}{l}\text { Estado } \\
\text { provincial (1) }\end{array}$ & 0,45 & 5 & 0 & 0 & 0 & 0 & 0 & 0 & 0 & 0,36 & 5 & 0 \\
\hline $\begin{array}{l}\text { Particulares } \\
\text { (6) }\end{array}$ & 3,91 & 23 & 0 & 2,5 & 5 & 0 & 20 & 20 & 20 & 4,86 & 23 & 0 \\
\hline
\end{tabular}

Fuente: Elaboración propia sobre la base de encuestas de campo (2007).

Nota: Acompaña a cada fuente de financiamiento, y entre paréntesis, el número de instituciones con fondos provenientes de cada una de ellas.

Al analizar el financiamiento de los establecimientos entrevistados de acuerdo a la pertenencia de algún tipo de asociación, se verifica que la Asociación de Clínicas del Interior es la que concentra la mayor cantidad de prestadores que alcanzaron acuerdos con PAMI, mientras que, un sólo prestador de la ACLISASAy uno de la Cámara de Empresas Médicas brindan servicios a esa institución. Por su parte, la Obra Social provincial, las Obras Sociales nacionales y las prepagas financian a prestadores de todas las asociaciones incluso a aquellos prestadores no asociados. La Tabla 6 presenta por agrupación intermedia y para el total, las fuentes de fondos sanatoriales

Cuadro 6 - Participación relativa (en \%) de los fondos de aseguramiento en el financiamiento de prestadores agrupados por asociación

\begin{tabular}{|c|c|c|c|c|c|c|c|c|c|c|c|c|c|c|c|c|}
\hline \multirow[t]{2}{*}{ Financiedoros } & \multicolumn{4}{|c|}{ ACLEASA (5) } & \multicolumn{4}{|c|}{ 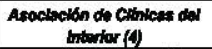 } & \multicolumn{4}{|c|}{$\begin{array}{l}\text { Cinem do emprease: } \\
\text { medticas (o) }\end{array}$} & \multicolumn{4}{|c|}{ Ahomene (앙 } \\
\hline & Prated & Inade & 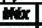 & 祡 & Petras & Ending & $\ln x$ & In & Prates & Inode & Sax & 简 & Faten & Exen & $\ln x$ & I典 \\
\hline PALAB & 1 & 85 & 85 & 85 & 4 & 39 & 80 & 20 & 1 & 15 & 15 & 15 & & & & \\
\hline Chre Soctal Pelel (14) & 4 & 34 & 50 & 5 & 4 & 28 & 40 & 10 & 3 & 40 & 75 & 20 & 3 & 30 & 34 & 25 \\
\hline Ouras Sochalas Neclonilas (1s) & 4 & 34 & 55 & 5 & 4 & 24 & 30 & 10 & 2 & 37,5 & 55 & 20 & 3 & 33 & 50 & 15 \\
\hline Preperan (10) & 3 & 7,6 & 10 & 5 & 1 & 5 & 5 & 5 & 3 & 28 & 55 & 5 & 3 & 29 & 33 & 25 \\
\hline Exdado provincled (t) & 1 & 5 & 5 & 5 & & & & & & & & & & & & \\
\hline Partilouleres (6) & 1 & 5 & 5 & 5 & 3 & 12 & 5 & 20 & 1 & 5 & 5 & 5 & 1 & 23 & 23 & 23 \\
\hline
\end{tabular}

Fuente: Elaboración propia sobre la base de encuestas de campo (2007). 
Los datos relevados sugieren que PAMI cuenta con un menor peso relativo que la Obra Social provincial. Asimismo las Obras Sociales nacionales destacan su relevancia en el financiamiento a los prestadores privados. A pesar de que el nivel de cobertura formal en la provincia es relativamente bajo (cercano al 40\%), esta situación podria estar dada por la diversificación do la actividad productiva y la presencia de Obras Sociales por rama de actividad. Un elemento que avala esta hipótesis es el aumento significativo del gasto de las Obras Sociales durante el período 1993-2004. De acuerdo con datos de la Dirección Nacional de Coordinación Fiscal con las Provincias, el Gasto en atención pública pasó del $74,8 \%$ del total de gasto en salud en 1993 al $56,7 \%$ en 2002 , mientras que el Gasto en atención a través de Obras Sociales aumentó desde el $25,2 \%$ al $43,3 \%$. Actualmente, la provincia de Salta gasta más que el promedio nacional en atención a través de Obras Sociales, y menos que el promedio en atención pública.

\section{CONTRATOS}

De acuerdo con los establecimientos entrevistados, PAMI brinda asistencia a través de seis establecimientos: tres en la ciudad de Salta, dos en el departamento de San Martin y uno en el de Anta. Sin embargo, cuatro de ellos mencionaron que desde que el PAMI dejó de ser gerenciado a través de instituciones intermedias, no poseen contratos sino actas acuerdo o cartas de intención que se renuevan cada 30 días. No obstante, tres son negociados directamente entre el prestador $\mathrm{y}$ el financiador $\mathrm{y}$ otros tres aún se canalizan vía Asociación de establecimientos.

Respecto a IPS, en su mayoría los contratos son negociados mediante Asociaciones de Establecimientos. De acuerdo con los datos relevados, en la ciudad de Salta, ocho establecimientos lo hacen via Asociación de Clinicas, tres lo hacen directamente y tres a pesar de estar dentro de la Cámara de Empresas Médicas de Salta, celebran contratos directos. En San Martín dos prestadores mantienen contrato con el IPS y la Asociación de Clínicas del Interior, uno negocia los contratos vía asociación y otro lo hace directamente.

Los convenios con la Obra Social provincial se firman de acuerdo a una tasa de uso ${ }^{6}$. Para ello, la provincia se encuentra dividida en tres zonas con las cuales

"Se firma por un año y se evalua si supera o no el techo presupuestario acordado a partir del cual se ajusta el monto para el año siguiente. 
elIPS firma convenios por Asociación: Salta Capital y zona de los Valles, la Cámara del Sur (Metan, Rosario de la Frontera) y la Cámara del Norte. El mecanismo de negociación de las Obras Sociales sindicales se asemeja al del IPS. En total, un 62\% de los contratos se realizan a través de alguna Asociación intermedia. En la ciudad de Salta se canaliza mayoritariamente vía Asociación de Clínicas, mientras que en el interior se divide entre la Asociación de Clínicas del Interior, contratación directa - mediante otros intermediarios. La información recogida sugiere que las asociaciones de clínicas, a pesar de su atomización, se constituyen en actores relevantes para la negociación de contratos.

Cuadro 7 - Mecanismos de negociación de los contratos

\begin{tabular}{|l|c|c|c|}
\hline \multirow{2}{*}{$\begin{array}{l}\text { Mecanismos de } \\
\text { Negociación de contratos }\end{array}$} & \multicolumn{3}{|c|}{$\begin{array}{c}\text { Fondos de } \\
\text { Aseguramiento }\end{array}$} \\
\cline { 2 - 4 } & PAMI & OSP & Otro \\
\hline Directa con el financiador & $60 \%$ & $23 \%$ & $38 \%$ \\
\hline Concurso de prestadores & & & \\
\hline $\begin{array}{l}\text { Vía Asociación de } \\
\text { establecimientos }\end{array}$ & $40 \%$ & $77 \%$ & $54 \%$ \\
\hline $\begin{array}{l}\text { Vía Asociación de } \\
\text { profesionales }\end{array}$ & & & \\
\hline Vía otro intermediario & & & $8 \%$ \\
\hline
\end{tabular}

Fuente: Elaboración propia sobre la base de encuestas de campo (2007).

Si bien el "Nuevo Modelo Prestacional" (NMP) de PAMI supone la contratación directa de prestadores, un $40 \%$ de ellos mantiene vinculación a través de un intermediario. Esta situación es posible a partir de una cláusula dentro del NMP que permite conformar redes para la atención. Tal es el caso de la red de la Asociación de Clínicas del Interior, quien administra las cápitas, las distribuye entre los prestadores y deriva pacientes a los prestadores de la capital provincial cuando ellas no pueden ser cubiertas por la oferta local. Respecto al plazo de vigencia de los contratos, en su mayoría, son renovados automáticamente incluyendo los cambios de precios mediante un acta modificatoria del convenio. Sólo con OSDE se registraron contratos con plazos establecidos entre seis meses y un año, y entre uno y dos años. 
Cuadro 8 - Mecanismos de pago

\begin{tabular}{|l|r|r|r|}
\hline $\begin{array}{l}\text { Mecanismos de } \\
\text { pago }\end{array}$ & PAMI & OSP & Otro \\
\hline Cápita & $100 \%$ & $7 \%$ & $17 \%$ \\
\hline Módulo & & $7 \%$ & $7 \%$ \\
\hline Prestación & & $93 \%$ & $77 \%$ \\
\hline Monto Fijo & & & $7 \%$ \\
\hline Cartera fija & & $7 \%$ & $7 \%$ \\
\hline
\end{tabular}

Fuente: Elaboración propia sobre la base de encuestas de campo (2007).

En todos los casos el PAMI paga a sus prestadores por cápita, lo cual implica una mayor transferencia de riesgo relativo aguas abajo. Sin embargo, se incorporan en los convenios una serie de estímulos complementarios. La tasa de incentivo es el más importante, que consiste en el pago adicional al prestador que supera un porcentaje de atención determinado. En el caso del médico de cabecera, superar el $70 \%$ del padrón asignado, recibe como base un adicional a la cápita de 15 pesos por paciente atendido, superando el umbral establecido. Existen estímulos similares en el caso de la internación y de las emergencias. Según las autoridades entrevistadas, éste es un mecanismo de ajuste a la subprestación que suelen generar los mecanismos de pago puramente capitados. Sin embargo, se registraron inconvenientes en el cobro de los mismos.

De acuerdo con los datos obtenidos en las entrevistas de campo, la cápita de PAMI incluye en el $40 \%$ de los casos baja complejidad, en el $100 \%$ media complejidad y sólo en el $20 \%$ alta complejidad, registrado en una clínica del interior provincial. El $40 \%$ de los que declararon trabajar con PAMI reciben una cápita de entre $\$ 11$ y $\$ 20$, mientras que el $60 \%$ restante declara precios de entre $\$ 21$ y $\$ 30$. Asimismo, existen extracápitas que requieren de autorización previa.

La modalidad de pago del IPS es por prestación con techo presupuestario. En abril de 2002 se declaró la emergencia sanitaria en el IPS a través del decreto provincial 560/02 y se modificó la modalidad de contratación y pago. Se firmaron convenios por los cuales se mantuvo el pago por prestación pero con techo financiero, que continúa en vigencia. 
Los mecanismos de pago utilizados por prepagas y Obras Sociales Nacionales no resultan tan homogéneos. Si bien el pago por prestación prevalece en el $77 \%$ de los casos, tanto OSPRERA como OSECAC impulsan pagos capitados, existiendo modelos modulados y de cartera fija.

Gráfico 7 - Distribución del PAMI y el IPS según tamaño de sus prestadores

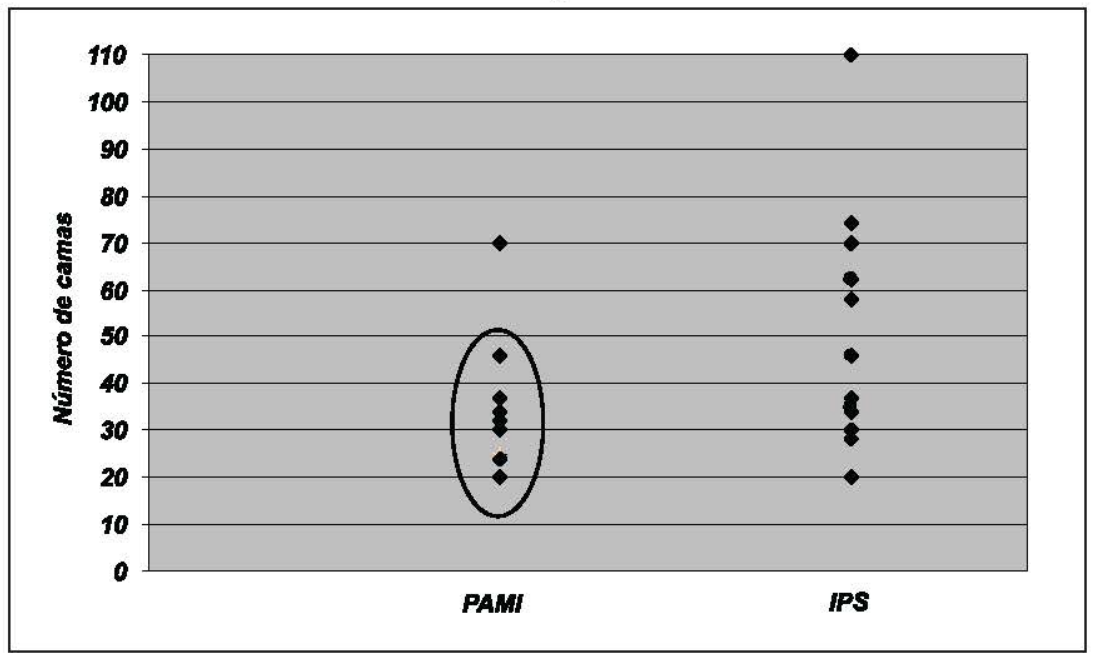

Fuente: Elaboración propia sobre la base de encuestas de campo (2007).

Entre otras diferencias significativas en la distinción de contratos, se observa que, PAMI contrata clínicas más pequeñas, medidas en términos de camas. Asimismo el pago de coseguros por parte de los afiliados no es permitido por PAMI, en tanto que el IPS cobra el $20 \%$ del valor de la prestación, luego descontado por la Obra Social al sanatorio sobre el total de la facturación. Respecto a las Obras Sociales sindicales, casi la mitad de los sanatorios entrevistados afirmó que se cobran coseguros.

Los mecanismos de pago a los médicos generales y a especialistas son por prestación en todos los casos, como surge de la lectura del Cuadro 9. 
Cuadro 9 - Pago de honorarios a médicos generales

\begin{tabular}{|l|r|r|r|}
\hline $\begin{array}{l}\text { Pago a médicos } \\
\text { generales }\end{array}$ & PAMI & OSP & Otro \\
\hline Intermediario & & $50 \%$ & $22 \%$ \\
\hline Establecimiento & $80 \%$ & & $42 \%$ \\
\hline Financiador & & $29 \%$ & $14 \%$ \\
\hline Otro: mixto & $20 \%$ & $21 \%$ & $22 \%$ \\
\hline
\end{tabular}

Fuente: Elaboración propia sobre la base de encuestas de campo (2007).

Sin embargo, existen diferencias significativas en cuanto quién realiza los pagos. Para el caso del PAMI, en el $80 \%$ de los casos el pago a profesionales se canaliza a través del establecimiento prestador y sólo en un caso se registró un sistema mixto. Respecto a la Obra Social Provincial, en el 50\% de casos la distribución de retribuciones la realizan las Entidades Intermedias (Asociación de Clínicas o Círculo Médico), en el $29 \%$ paga el propio financiador y un $21 \%$ se realiza en forma mixta. En ningún caso el establecimiento se encuentra directamente a cargo de la retribución al profesional.

Finalmente, en relación a los médicos que prestan servicios a beneficiarios de Obras Sociales Sindicales, en el $42 \%$ de los casos paga directamente el establecimiento, en el $22 \%$ algún intermediario, en el $14 \%$ el financiador y en otro $22 \%$ se establece un mecanismo mixto. Esta variedad de mecanismos refieren al diferencial de poder relativo de cada institución intermedia (institucional y profesional) en función del organismo pagador y de la localización del servicio.

Cuadro 10 - Pago de honorarios a médicos especialistas

\begin{tabular}{|l|c|c|c|}
\hline $\begin{array}{l}\text { Médicos } \\
\text { especialistas }\end{array}$ & PAMI & OSP & Otro \\
\hline Intermediario & & $58 \%$ & $28 \%$ \\
\hline Establecimiento & $80 \%$ & & $42 \%$ \\
\hline Financiador & & $21 \%$ & $15 \%$ \\
\hline Otro & $20 \%$ & $21 \%$ & $15 \%$ \\
\hline
\end{tabular}

Fuente: Elaboración propia sobre la base de encuestas de campo (2007). 
El pago a los médicos especialistas se distribuye de forma similar a los generales. Sin embargo se presenta una diferencia respecto a la Obra Social Provincial, con quien el Círculo Médico se constituye en el principal pagador, contando con dos padrones de profesionales diferenciados por antigüedad.

\section{SUBSECTOR PUBLICO}

El subsector público posee una dinámica con caracteristicas diferentes al privado vinculado no sólo a su objetivo social sino a las particularidades y restricciones vinculadas a su gestion. Su estructura no se encuentra determinada por el nivel de concentración de las instituciones prestadoras, ni por la competencia en la atracción de pacientes, siendo la negociación de contratos y fijación de precios con los financiadores temáticas de segundo orden.

Su principal y casi exclusivo financiador es el Estado provincial, que asigna anualmente un presupuesto destinado a brindar servicios a quienes acuden en búsqueda de atención y mayoritariamente no poseen cobertura formal. Ello se realiza por medio de su red de prestadores (hospitales, centros de salud, postas sanitarias, etc.). El Estado nacional participa de este proceso mediante la descentralización de recursos (monetarios o en especie) canalizados a través de programas tales como el Plan Nacer, Remediar, entre otros. Finalmente, las Obras Sociales y el PAMI, en considerable menor medida, participan del financiamiento de los hospitales públicos configurando un esquema de autogestión que varía de acuerdo al porcentaje de facturación y cobro efectivo a dichas instituciones.

Para el análisis de esta sección se entrevistaron seis hospitales públicos con características diferentes marcadas por su grado de descentralización en la recaudacion de recursos:

El Hospital Castellanos (Gral. Quemes-Anta), Hospital El Milagro (Ciudad de Salta) y Hospital San Roque (Embarcación-Anta) y el Nuevo Hospital de Tartagal (Tartagal-San Martín), de gestión fundamentalmente centralizada; el Hospital San Bernardo (Ciudad de Salta) de gestion descentralizada; Hospital Ntra. Sra. del Rosario (Cafayate) de gestión conjunta con una organización no gubernamental y el Hospital El Nuevo Milagro de gestión privada, localizado en la capital provincial. 
Se aplicó un cuestionario orientado fundamentalmente a evaluar la distribución del financiamiento, la capacidad de toma de decisiones relacionadas, con la participación de los directivos en la formulación del presupuesto, la contratación de personal, el establecimiento de mecanismos de incentivos al personal, y la compra de insumos.

\section{VII.1. Estructura del subsector público}

La Constitución de la provincia de Salta, en su preámbulo, menciona expresamente el derecho a la vida, y en particular a la salud. Destacando la intangibilidad de la integridad fisica y moral de la persona. Mediante el art. 41, la salud es reconocida como un bien social. Si bien conforma un derecho inherente a la vida, presenta un doble rol que otorga a la persona el deber de preservarlo. Compete al Estado el cuidado de la salud física, mental y social de las personas, y asegurar a todos la igualdad de prestaciones ante idénticas necesidades.

El Estado debe elaborar el Plan de Salud Provincial con la participación de los sectores socialmente interesados, contemplando las distintas etapas de promoción, prevención, restauración y rehabilitación de la salud, introduciendo el art. 42 el concepto de "justicia social" y "utilización racional de los recursos" como pilares de la acción estatal. El derecho a la salud también es mencionado entre los derechos que se reconocen al trabajador.

En el articulo 31 se profundiza sobre la relación de consumo, estableciendo no solamente que los ciudadanos tienen derecho a la protección de su salud y seguridad, sino que además el Estado deberá defender activamente la competencia, evitando toda forma de "distorsión de los mercados, el control de los monopolios naturales y legales, la calidad y eficiencia de los servicios públicos y la constitución de asociaciones de consumidores y usuarios...". En particular, estipula que la publicidad deberá evitar inducir a conductas adictivas o perjudiciales o promover la automedicación y establece sanciones contra los mensajes que distorsionen la voluntad de compra del consumidor mediante técnicas que la ley determine como inadecuadas.

En relación a la via idónea para solicitar la efectivización de las garantas constitucionalmente reconocidas, como es el derecho a la salud, el art. 87 reconoce la acción de amparo, que procede frente a cualquier decisión, acto u omisión 
arbitrarios o ilegales de la autoridad, excepto la judicial, o de particulares, restrictivos o negatorios de las garantías y derechos subjetivos explícitos o implícitos. En particular, existe un importantísimo reconocimiento: la propia Carta Magna establece que la no prestación injustificada por parte del Estado de los servicios educativos, de salud y de otros esenciales - es decir la sola no prestación, también llamada falta de servicio público- da lugar a esta acción. Además de la Constitución provincial, existen una serie de normativas que dan forma al sistema de salud y operacionalizan estos derechos, como ser la Resolucion Ministerial 370 de 1985, que organiza el sistema público de provisión de servicios de salud, definiendo los niveles de atención, regionalización y descentralización de las áreas operativas.

Por su parte, la ley 6841 , promulgada en 1996, que convirtio en ley al Decreto 68/95, consagró los principios sobre el Plan de Salud Provincial que según sus previsiones, las prestaciones establecidas en el marco normativo serán consideradas rninimas, deberán asegurar la plena, eficaz y eficiente utilización de los servicios y capacidad instalada, y estarán basadas en la estrategia de la atención primaria de la salud y en el derecho del habitante a la libre elección del profesional, con las limitaciones propias del manejo eficiente de los recursos. Asimismo, esta ley establece la reestructuración de los hospitales públicos incorporando la figura del "Hospital de Autogestión". Las principales atribuciones que poseen estas instituciones son:

-Realizar convenios con entidades de la seguridad social provinciales y nacionales y, muy especialmente, con las obras sociales de jurisdicción nacional.

-Complementar servicios con otros establecimientos asistenciales públicos o privados.

-Cobrar los servicios que brinden a través de los mecanismos del seguro de salud a cargo del Instituto Provincial de Salud o a las personas con capacidad de pago o a terceros pagadores que cubran las prestaciones del usuario de obras sociales, mutuales, empresas de medicina prepaga, seguros de accidente, medicina laboral, convenios celebrados por el Gobernador con los gobiernos de las provincias vecinas o de los países limítrofes, en el marco del artículo 124 de la Constitución nacional, a los fines de la contribución de dichos gobiemos, para la financiación de las prestaciones destinadas a satisfacer las necesidades de los oriundos de unas y otros, mientras no se radiquen en la provincia de conformidad con el ordenamiento, y otros similares.

- Integrar redes de servicios de salud con otros establecimientos asistenciales 
públicos y privados de la provincia, de la región del Noroeste argentino o de la Nación, debidamente habilitadas.

Más allá de su capacidad recaudatoria, los hospitales de autogestión continúan recibiendo los ingresos presupuestarios que le asigna la pertinente ley para el habitual funcionamiento del mismo.

La Superintendencia de Servicios de Salud, de competencia nacional, es quien fiscaliza el cumplimiento de las obligaciones de las Obras Sociales respecto de los pagos por las prestaciones efectuadas a sus beneficiarios por los Hospitales Públicos de Gestión Descentralizada, procediendo a realizar el débito automático. Si correspondiere y acorde a la normativa, la Superintendencia opera un sistema de débito automático por medio del cual la AFIP descuenta directamente de la recaudación de la Obra Social involucrada el importe de la deuda conformada con el Hospital.

Finalmente, la ley 6841 también da origen a la implementación de un sistema de Seguro de Salud que financie las prestaciones contempladas en el marco normativo, las que estarån a cargo de prestadores privados y prestadores públicos. Dicho Seguro se encuentra en funcionamiento en algunas áreas y está orientado a brindar cobertura sanitaria a la población sin cobertura de obra social y sin capacidad de pago. Mediante el pago de una cápita por habitante sin cobertura formal, el Estado garantiza la atención en todo el territorio de la provincia incluyendo derivaciones ante eventualidades.

En la actualidad al sistema se encuentra organizado cuatro regiones sanitarias que a su vez contienen redes de atención.

(i) Norte: red de atención Orán y de red de atención Tartagal

(ii) Sur: red de atención Gral. Quemes y red de atención Metăn

(iii) Centro: red de atención Noroeste y red de atención Sureste

(iv) Oeste: red de atención Cachi, red de atención Cafayate y red de atención Valle de Lerma y Puna.

Las redes de atención están conformadas por establecimientos sanitarios de niveles de atención y complejidad creciente. Se formaron bajo cl supuesto de que mejoran el acceso a los servicios de salud, la calidad de la atención sanitaria, optimizan la utilización de recursos y generan eficiencia en la prestación de 
servicios de salud. Además facilitan la articulación de las unidades prestadoras, la utilización adecuada de la oferta, la racionalizan los costos y optimizan de la infraestructura.

Las redes tienen una población definida y a su vez están conformadas por áreas operativas. Esto implica que, en general, el paciente ingresa al sistema de salud a través del centro de atención primaria y puede ser derivado a otro centro $u$ hospital de mayor complejidad. Cada red de atención está conformada por un hospital de referencia y otros establecimientos de distintos niveles de complejidad. En total, Salta cuenta con 50 hospitales publicos ( 35 de nivel 2,8 de nivel 3 y 7 de nivel 4) y 467 centros de salud y puestos sanitarios articulados en distintos niveles de atención:

-El primer nivel de atención está formado por puestos fijos (no cuentan con personal sanitario permanente); puestos sanitarios (cuentan con atención de enfermería permanente) y centros de salud (con o sin internación y ofrecen atención médica y de enfermeria diaria y de otros profesionales de la salud en forma permanente o programada. Pueden tener atención odontológica diaria y otros servicios como laboratorio, radiologia etc.)

-El segundo nivel de atención está constituido por servicios de salud con atención ambulatoria y de internación brindados por médicos generalistas. Se dispone de odontología y servicios de diagnóstico y tratamiento como bioquímica, radiología y en algunos casos tienen guardias activas o pasivas y atención especializada en forma periódica.

-Conforman el tercer nivel de atención los establecimientos de salud con atención ambulatoria y de internación, con especialidades básicas, además de odontología, bioquímica, radiologia, farmacia, hemoterapia, anestesiología, quirófanos con resolución de especialidades traumatologicas y quirúrgicas de bajo y mediano riesgo.

-Finalmente, cuarto nivel de atención está representado por hospitales de alta complejidad que permiten resolver prácticamente la totalidad de los problemas de salud de la poblacion. Son establecimientos de referencia en las regiones sanitarias y, algunas ocasiones lo son de la jurisdicción provincial o región.

\section{VII.2. Distribución del financiamiento}

De acuerdo a la información relevada mediante las entrevistas y encuestas a prestadores, el financiador más destacado del sistema público es el propio Estado 
provincial. De acuerdo al grado de descentralización en la toma de decisiones y al nivel de autogestión aumenta la participación de los seguros (PAMI, obro sociales sindicales) y particulares en el financiamiento institucional. A continuación se presenta la Tabla 13 que caracteriza tres modelos de gestión donde se pone de manifiesto diferenciales de gerenciamiento de los hospitales salteños.

Si bien el hospital cogestionado por una organización no gubernamental obtiene casi el $50 \%$ de su financiamiento del Estado provincial, el otro $50 \%$ se distribuye entre el PAMI, Plan Nacer (Estado nacional) y en menor medida las obras sociales nacionales. En el hospital descentralizado, el Estado provincial es claramente el mayor aportante, participando en un $90 \%$ en el presupuesto y en menor medida los demás financiadores. Se observa que el hospital de gestión centralizada, es el Estado provincial el principal financiador.

La participación de las Obras Sociales nacionales y el PAMI es significativamente reducida. A esto se debe agregar que, en general, los hospitales públicos no celebran convenios con las primeras, ya que la Superintendencia de Servicios de Salud actúa como intermediario en el cobro. En este sentido, no existe posibilidad de negociar precios ni mejores condiciones para la prestación de servicios.

Cuadro11 - Distribución del financiamiento por tipo de gestión

\begin{tabular}{|l|r|r|r|r|r|r|}
\hline \multicolumn{1}{|c|}{$\begin{array}{c}\text { Tipo de } \\
\text { hospital }\end{array}$} & PAMI & $\begin{array}{l}\text { Estado } \\
\text { Pcial }\end{array}$ & OSN/IPS & Part. & Profe. & $\begin{array}{l}\text { Plan } \\
\text { Nacer }\end{array}$ \\
\hline $\begin{array}{l}\text { Cogestionado } \\
\text { con ONG }\end{array}$ & $27 \%$ & $49 \%$ & $4 \%$ & $2 \%$ & & $18 \%$ \\
\hline Descentralizado & $1 \%$ & $88 \%$ & $10 \%$ & & $1 \%$ & $0 \%$ \\
\hline Centralizado & & $95 \%$ & $1,50 \%$ & & & $3,50 \%$ \\
\hline
\end{tabular}

Fuente: Elaboración propia sobre la base de encuestas de campo (2007)

Aunque en promedio un $25 \%$ de los pacientes que atienden los hospitales públicos posee algún tipo de cobertura, los montos de la autogestión no impactan significativamente en los presupuestos hospitalarios. Se factura en promedio sólo un $8 \%$ del presupuesto total y se cobra un $5 \%$. Adicionalmente, el $20 \%$ de lo efectivamente cobrado es transferido al nivel central y el resto permanece en los hospitales, pudiéndose aplicar estos recursos a la compra de insumos, contratación 
de personal, etc.

VII.3. Participación en la toma de decisiones

De acuerdo con los datos relevados, la mayor parte de las decisiones se toman a nivel provincial desde el Ministerio de Salud. De las instituciones entrevistadas sólo dos afirmaron tener la capacidad de proponer el presupuesto hospitalario, lo cual ratifica la centralización del Estado provincial. Asimismo, la atribución de contratar recursos humanos de planta también es responsabilidad del Ministerio de Salud provincial. La modalidad de pago más frecuente es el salario. Los profesionales de la salud y demás personal reciben una remuneración en función de la cantidad de horas contratadas. En general, el salario no guarda relación con el nimero de consultas y la cantidad de pacientes. Tampoco generan incentivos directos a prestar particular atención a la calidad del servicio provisto o a la satisfacción del paciente.

No obstante, los hospitales de autogestión tienen capacidad de contratar personal con los fondos de autogestion o del Plan Nacer en la mitad de los casos. El hospital Nuestra Señora del Rosario, que cogestiona los fondos públicos (Seguro Provincial de Salud) con una organización no gubernamental, requiere acordar las contrataciones y compras en las que se utilicen dichos fondos. EINuevo Hospital EI Milagro, de gestion privada y el hospital San Bernardo, de gestion descentralizada son los únicos que establecen mecanismos de incentivos a su personal con los fondos de la autogestión'. En estos casos el Ministerio aún efectúa las compras de insumos de alta complejidad o inversiones en infraestructura. En el resto de las operaciones de abastecimiento se recurre a licitaciones a nivel hospitalario, conformándose comités de compras.

\footnotetext{
'Se realizan convenios de gestión para cada servicio donde se establecen objetivos de producción y calidad. Se hace seguimiento de objetivos mensuales y a partir de ellos se registran los desvios de producción y calidad. Si los desvios están dentro de 10 s $+5 \%$ se otorga un pago complementario.
} 
Cuadro 12 - Responsabilidad en la toma de decisiones por tipo de decisor

\begin{tabular}{|l|l|l|l|}
\hline \multicolumn{1}{|c|}{ Decisor } & Presupuesto & RRHH & Compras \\
\hline Ministerio (provincial) & $3 / 6$ & $6 / 6$ & $5 / 6$ \\
\hline Secretaria (municipal) & $0 / 6$ & $0 / 6$ & $0 / 6$ \\
\hline Director & $0 / 6$ & $3 / 6$ & $4 / 6$ \\
\hline Director con Ministerio & $2 / 6$ & $1 / 6$ & $1 / 6$ \\
\hline Director con ONG & $0 / 6$ & $1 / 6$ & $1 / 6$ \\
\hline
\end{tabular}

Fuente: Elaboración propia sobre la base de encuestas de campo (2007).

Cuadro 13 - Responsabilidad en la toma de decisiones (por tipo de prestador)

\begin{tabular}{|l|c|c|c|c|}
\hline $\begin{array}{l}\text { Tipo de } \\
\text { institución }\end{array}$ & Presupuesto & RRHH & Compras & Subcontrataciones \\
\hline $\begin{array}{l}\text { Cogestionado } \\
\text { con ONG }\end{array}$ & $\mathrm{si}$ & $\mathrm{si}$ & $\mathrm{si}$ & $\mathrm{si}$ \\
\hline Descentralizado & $\mathrm{si}$ & $\mathrm{si}$ & $\mathrm{si}$ & $\mathrm{si}$ \\
\hline Centralizado & no & no & si & no \\
\hline
\end{tabular}

Fuente: Elaboración propia sobre la base de encuestas de campo (2007)

Los Cuadros 12 y 13 resumen las atribuciones y responsabilidades en la gestión hospitalaria para tres indicadores claves (definición del presupuesto, contratación de personal y compras) por tipo de decisor y por características de la institución prestadora.

\section{CONCLUSIONES}

El objetivo del trabajo ha sido analizar el sistema de salud de la provincia de Salta, los mecanismos de contratación y de pago, y sus resultados en términos de transferencia y absorción de riesgos entre financiadores y prestadores sanitarios. Para ello se indagó sobre las características de la estructura del mercado privado de salud de la provincia (número de competidores y concentración de la oferta); el grado de competencia y diferenciación de producto entre los proveedores, y sus 
estrategias para captar a sus principales (financiadores y pacientes); las características de los contratos y los mecanismos de pago, el peso relativo de los actores en las negociaciones para establecerlos y finalmente los modos de transferencia y absorción de riesgo entre aseguradores y prestadores.

Respecto del sector público, el análisis se orientó en torno al nivel de descentralización en la toma de decisiones y la capacidad del sector ante los incentivos monctarios desplegados por los fondos de aseguramiento social. A partir de los interrogantes planteados y de los resultados obtenidos por el estudio se arribaron a las siguientes conclusiones.

La estructura del mercado privado de la provincia de Salta presenta marcadas diferencias entre la capital y el interior. Se comprueba el supuesto que mercados más desarrollados en términos de escala poblacional generan un escenario de mayor nivel de competencia entre prestadores y la consecuente pérdida de poder relativo del principal competidor. Asimismo, bajo condiciones de mayor competencia se desarrollan mayores incentivos a diferenciarse mediante estrategias alternativas a los precios. Reputación, hotelería e infraestructura son los principales motivos de competencia entre los prestadores privados.

Por otra parte se registró cierta divergencia de intereses entre los principales (pacientes y financiadores) y por ende estrategias diferenciadas desarrolladas por los agentes (prestadores). La capacitación de los recursos humanos $(93 \%)$, la hotelería (67\%) y la incorporación de tecnología (53\%) son las estrategias más consideradas por las entidades encuestadas al momento de atraer a los pacientes. La tecnología (60\%), la capacitación de los recursos humanos (47\%) y la hotelería ( $47 \%$ ) fueron los motivos más valorados al momento de identificar la causa de elección de los financiadores.

Caracterizada la estructura de mercado percibida por los prestadores del sistema de salud salteño, se avanzó en la distribución del financiamiento, los contratos y los mecanismos de pago, para identificar el poder de mercado y capacidad negociadora de cada actor participante. La información recogida muestra que la Obra Social provincial, las Obras Sociales nacionales y el PAMI respectivamente, son los principales financiadores del sector prestacional privado. No obstante, se registran marcadas diferencias entre la ciudad capital y el interior, donde el PAMI tiene mayor participación. Comparativamente con otros financiadores, se comprobó que PAMI contrata clínicas más pequeñas, medidas en 
términos de camas, a las cuales le puede transferir el riesgo mediante un mecanismo de pago capitado.

Contrariamente, la Obra Social Provincial, con mayor presencia en los centros urbanos, contrata prestadores de mayor envergadura en donde el riesgo es compartido entre el financiador y el prestador mediante pagos por prestación con techo financiero. Finalmente, se destaca la presencia de las Obras Sociales Nacionales aportando cerca del $40 \%$ del financiamiento al subsistema privado. Excepto para la definición de contratos con PAMI, las asociaciones de clínicas tanto de capital como del interior se constituyen en actores relevantes para la estructuración de vínculos contractuales entre seguros sociales y privados y las clínicas polivalentes salteñas.

Por su parte, el subsector público posee una dinámica con características diferentes al privado vinculado no sólo a su objetivo social sino a las particularidades y restricciones en su gestión. Su estructura no se encuentra determinada por el nivel de concentración de las instituciones prestadoras, ni por la competencia en la atracción de pacientes, siendo la negociación de contratos y fijación de precios con los financiadores temáticas de segundo orden. El financiador más destacado del sistema público es el propio Estado provincial. No obstante, el caso salteño presenta cierta variedad de sistemas de gestión que se extienden desde hospitales altamente centralizados en el Ministerio de Salud provincial, hasta otros con participación de organizaciones no gubernamentales. En este sentido, el grado de descentralización en la toma de decisiones varia a medida que se registra un mayor nivel de autogestion medido en términos de participación de otros financiadores distintos al Estado provincial.

\section{REFERENCIAS BIBLIOGRAFICAS}

Chawla, M. et al., (1997), Paying the Physician: Review of Different Methods, Department of Population and International Health, Harvard School of Public Health, Boston, Massachusetts.

Chernichovsky, D., Bolotin, A. y De Leeuw, D. A Fuzzy (2003), Logic Approach Toward Solving the Analytic Enigma of Health System Financing, The European Journal of Health Economics, Vol. 4, (3).pp. 158-175.

Hart, O. y Holmstrom, B.s (1987) The Theory of Contracts, en Advances in 
Economic Theory, Fifth World Congress, ed. T. Bewley. Cambridge University, Press.

Langenbrunner, J. y Xingzhu La, (2004), How to Pay? Understanding and Using Incentives, The Intermational Bank for Reconstruction and Development, The World Bank.

Llaya, J., (2007), Actores y Agenda en el Sistema de Salud Argentino, Ministerios de Salud Provinciales: El Caso de Salta, Serie Seminarios Salud y Política Pública, CEDES, WPN4, Buenos Aires, Argentina, Agosto.

Maceira, D., (2006), Descentralización y Equidad en el Sistema de Salud Argentino, en Flood, C., "La política del gasto social: lecciones de la experiencia argentina", Editorial La Colmena, Buenos Aires, Argentina.

Maceira, D., (2010), Financiamiento, Aseguramiento y Prestación de Servicios de Salud en Argentina, en Sistemas de Salud Comparados en América Latina, Knaul, F. (coordinadora), DRC, en prensa.

Maccira, D., (2008), Crisis Económica, Politica Pública y Gasto en Salud: La Experiencia Argentina. CIPPEC, Buenos Aires, Enero 2008.

Maceira, D. y Poblete, $C_{\text {s }}$ (2003), Estructura de mercado y mecanismos de pago en salud: Experiencias en la provincia de Mendoza, Argentina, 1989-1999, Desarrollo Económico, Vol.43, (171), oct-dic.

Maceira, D., (1998), Provider Payment Mechanisms in Health Care: Incentives, Outcomes, and Opganizational Impact in Developing Countries, Partnerships for Health Reform. Primers for Policymaking, Bethesda, Maryland, Abt Associates Inc.

Milgrom, P. y Roberts, J., (1992), Economics, Organization and Management, Englewood Cliffs, EUA. Prentice-Hall.

Ministerio de Salud de la Nación,(2000), Guía de Establecimientos de Salud. Trabajo publicado en: www.deis.gov.ar, Buenos Aires, Argentina.

Petersen, L. et al., (2006), Does Pay-for-Performance Improve the Quality of Health Care?, Annals of Internal Medicine. Agosto.

Prats J. y Zeckhauser R., (1992), Principals and Agents, Boston, Harvard Bussines School Press, 1992.

Robinson, J., (2001), Theory and Practice in the Design of Physician Payment Incentives. The Milbank Quarterly, Vol. 79, (2), pp. 149-177.

Shen $Y .,(2003)$, Selection incentives in a performance-based contracting system. Health Services Research, Vol.38, (2), pp. 535-552, Abril. 